\title{
Withaferin A inhibits inflammatory responses induced by Fusobacterium nucleatum and Aggregatibacter actinomycetemcomitans in macrophages
}

\author{
EUI-JEONG NOH ${ }^{1}$, MING-JUNG KANG ${ }^{2}$, YU-JIN JEONG ${ }^{1}$, JUN-YOUNG LEE ${ }^{2}$, \\ JUNG-HWAN PARK ${ }^{1}$, HYE-JIN CHOI ${ }^{1}$, SANG-MUK OH ${ }^{1}$, KYUNG-BOK LEE ${ }^{1}$, \\ DONG-JAE KIM ${ }^{1}$, JI-AE SHIN $^{3}$, SUNG-DAE $\mathrm{CHO}^{3}$ and JONG-HWAN PARK ${ }^{2}$ \\ ${ }^{1}$ Department of Biochemistry, College of Medicine, Konyang University, Daejeon 302-718; \\ ${ }^{2}$ Laboratory of Animal Medicine, College of Veterinary Medicine and BK21 Project Team, Chonnam National University, \\ Gwangju 500-757; ${ }^{3}$ Department of Oral Pathology, School of Dentistry, Institute of Oral Bioscience, \\ Chonbuk National University, Jeonju, North Jeolla 561-756, Republic of Korea
}

Received March 23, 2015; Accepted April 26, 2016

DOI: $10.3892 / \mathrm{mmr} .2016 .5326$

\begin{abstract}
Periodontitis is a progressive chronic inflammatory disease and a major cause of tooth loss in humans. As a withanolides, withaferin A (WA) is known to exhibit strong anti-inflammatory activity. The present study examined whether WA inhibited inflammatory responses in macrophages in response to two representative periodontal pathogens, Fusobacterium nucleatum and Aggregatibacter actinomycetemcomitans. Murine bone marrow-derived macrophages (BMDMs) were used in this study and cytokine production in culture supernatants was measured by enzyme-linked immunosorbent assays. Western blot analysis was performed to determine the activation of nuclear factor $-\kappa B$ and mitogen-activated protein kinases (MAPKs) and the expression of inducible nitric oxide synthase (iNOS), toll-like receptor (TLR) 2 and TLR4. The production of nitric oxide (NO) was determined by the Griess reaction. WA treatment was shown to decrease interleukin (IL)-6 and tumor necrosis factor (TNF)- $\alpha$ production in BMDMs in response to $F$. nucleatum and $A$. actinomycetemcomitans in a dose-dependent manner. The phosphorylation of I $\mathrm{B}-\alpha$ and MAPKs (p38, extracellular signal-regulated kinases and c-Jun N-terminal kinases) induced by $F$. nucleatum and A. actinomycetemcomitans was also inhibited by WA. F. nucleatum and A. actinomycetemcomitans induced iNOS expression and NO production in BMDMs, which was inhibited by WA in a dose-dependent
\end{abstract}

Correspondence to: Professor Jong-Hwan Park, Laboratory of Animal Medicine, College of Veterinary Medicine and BK21 Project Team, Chonnam National University, 77 Yongbong-ro, Buk-gu, Gwangju 500-757, Republic of Korea

E-mail: jonpark@jnu.ac.kr

Key words: Withaferin A, inflammation, Fusobacterium nucleatum, Aggregatibacter actinomycetemcomitans, macrophages manner. WA also reduced endogenous and induced expression of TLR 2 and TLR4 in these cells. These results suggest that WA may be a potential therapeutic agent or preventive additive for periodontitis control.

\section{Introduction}

Diseases of the oral cavity include dental caries, periodontitis, cervical abrasion and halitosis (1). Periodontitis, a bacteria-induced chronic inflammatory disease, is recognized as the most common cause of tooth loss in humans $(2,3)$. Gram-negative bacteria, including Porphyromonas gingivalis, Prevotella intermedia, Fusobacterium nucleatum and Aggregatibacter actinomycetemcomitans are major pathogens involved in periodontitis. At the early stage of infection, these bacteria activate host innate immune responses, which result in the recruitment of neutrophils, macrophages and lymphocytes to the site of infection. The progression to chronic inflammation leads to the formation of a periodontal pocket, ulceration of the gingival surface, destruction of the periodontal ligament and the alveolar bone, and finally tooth loss $(4,5)$. Therefore, it is important to inhibit inflammation in periodontal tissues to maintain tooth health. Recently, there has been an increased interest in the field of herbal medicine as a source of novel therapeutic agents that exhibit anti-inflammatory properties for use in the treatment of periodontal diseases (6).

Withania somnifera is a member of the Solanaceae or nightshade family and its major components are alkaloids and withanolides. The latter consist of a steroid backbone bound to a lactone or one of its derivatives and exert prominent anti-inflammatory activities (7). Withaferin A (WA) is a representative withanolide and has been widely investigated for its anti-inflammatory and anti-cancer effects. A previous study demonstrated that WA reduces lipopolysaccharide (LPS)-induced inducible nitric oxide synthase (iNOS) expression and nitric oxide (NO) production by downregulating Akt and nuclear factor (NF) $-\kappa \mathrm{B}$ activation in a macrophage cell line (8). In addition, WA inhibits the constitutive or induced 
expression of inflammatory mediators, such as cytokines and adhesion molecules (such as intercellular adhesion molecule 1 and vascular cell adhesion protein), in various cells, including epithelial cells (9-12), suggesting that WA has anti-inflammatory effects in a wide range of host cells.

As representative innate immune cells, macrophages are specialized phagocytes and are responsible for the control of the growth of invading bacteria. They can produce inflammatory mediators, such as cytokines, chemokines or NO, via pattern recognition receptor- (PRR-) mediated signaling. Toll like receptors (TLRs) are a representative family of PRRs and are characterized by a cytosolic effector Toll/interleukin (IL)-1R homology (TIR) domain and extracellular leucine-rich repeats (LRRs) that are responsible for the recognition of microbial molecules (13). Several studies have shown that TLR2 and TLR4 are involved in cellular immune responses to periodontal pathogens (14-16). However, thus far the effect of WA on TLR expression in host cells remains unknown. In the present study, the inhibitory effect of WA was examined on the inflammatory responses of macrophages in response to two periodontal pathogens, F. nucleatum and A.actinomycetemcomitans.

\section{Materials and methods}

Animals. Wild-type male C57BL/6 mice (8 weeks old; 21-23 g body weight) were purchased from Koatech (Pyeongtaek, Korea). The animals were housed in an animal room at a constant temperature $\left(22-24^{\circ} \mathrm{C}\right)$ and light-dark cycle with $14 \mathrm{~h}$ of light and $10 \mathrm{~h}$ dark. Food and water were available ad libitum. Mice were acclimatized to the laboratory room for 1-3 weeks prior to the experiment. Mice were sacrificed by cervical dislocation and their femur and tibia were used to prepare macrophages. Animal studies were approved and conducted according to the regulations of the Institutional Animal Care and Use Committee at Konyang University (Daejeon, Korea).

Bacterial culture. F. nucleatum (25586; American Type Culture Collection, Mannasas, VA, USA) and A. actinomycetemcomitans (43718; American Type Culture Collection) were purchased from the American Type Culture Collection (Manassas, VA, USA). Bacteria were grown on brain heart infusion (BHI) broth containing hemin $(5 \mathrm{mg} / \mathrm{ml})$ and vitamin $\mathrm{K}$ $(10 \mathrm{mg} / \mathrm{ml})$ at $37^{\circ} \mathrm{C}$ under anaerobic conditions. Bacteria were allowed to grow to optical density (OD)600 $=0.6$, which corresponds to $\sim 10^{9} \mathrm{CFU} / \mathrm{ml}$ of viable bacteria as determined by serial dilution and plate counts, and frozen aliquots were stored at $-80^{\circ} \mathrm{C}$. For bacterial infection, aliquots were thawed and diluted to the desired concentration in phosphate-buffered saline or media.

Preparation and stimulation of murine macrophages. Bone marrow-derived macrophages (BMDMs) were prepared as previously described (17). Bone marrow cells were cultured with Iscove's modified Dulbecco's medium (IMDM; Welgene, Gyeongsan, Korea) containing 30\% L929 cell culture supernatant (KCTC, Jeongeup, Korea), 1X minimum essential medium, non-essential amino acids, $1 \mathrm{mM}$ sodium pyruvate, $10 \%$ fetal bovine serum and $100 \mathrm{U} / \mathrm{ml}$ penicillin/streptomycin (all purchased from Gibco; Thermo Fisher Scientific, Inc.,
Waltham, MA, USA) for 6 days. The cells were seeded in 48 -well plates at a concentration of $2 \times 10^{5}$ cells/well or in 6 -well plates at a concentration of $2 \times 10^{6}$ cells/well, and incubated in a $5 \% \mathrm{CO}_{2}$ incubator at $37^{\circ} \mathrm{C}$. The day after seeding, the cells were infected with $F$. nucleatum and A. actinomycetemcomitans at the indicated multiplicity of infection (MOI; presented as macrophage/bacterium ratios) in the absence or presence of WA (50-1,000 nM, Sigma-Aldrich, St. Louis, MO, USA). Culture supernatants were collected $6 \mathrm{~h}$ after infection for further analysis.

Measurement of cytokines. The concentrations of IL-6 and tumor necrosis factor (TNF)- $\alpha$ in culture supernatants from F. nucleatum- and A. actinomycetemcomitans-infected BMDMs were determined using a commercial enzyme-linked immunosorbent assay (ELISA) kit (R\&D Systems, Inc., Minneapolis, MN, USA).

Measurement of nitric oxide. The NO synthase activity in the culture supernatant of infected cells was determined by measuring the NO accumulation by the Griess reaction as previously described (18).

Immunoblotting. BMDMs were infected with $F$. nucleatum or A. actinomycetemcomitans at MOI 10 with or without pretreatment of WA $(250 \mathrm{nM})$ for $2 \mathrm{~h}$, and were lysed at the indicated time points $(0,15,30$ and $60 \mathrm{~min})$. The cells were lysed in a buffer containing $1 \%$ Nonidet-P40 supplemented with a complete protease inhibitor cocktail (Roche Diagnostics Deutschland GmbH, Mannheim, Germany) and $2 \mathrm{mM}$ dithiothreitol (Sigma-Aldrich). The extracted protein concentration was measured using a Bio-Rad Protein Assay Dye Reagent Concentrate (cat. no. 500-0006; Bio-Rad Laboratories, Inc., Hercules, CA, USA). Samples of protein $(30 \mu \mathrm{g})$ were cooled on ice following incubation at $95-100^{\circ} \mathrm{C}$ for $10 \mathrm{~min}$. Lysates were separated by $10 \%$ sodium dodecyl sulfate-polyacrylamide gel electrophoresis and transferred to polyvinylidene fluoride membranes by electroblotting. The membranes were blocked by incubation with $5 \%$ skimmed milk for $1 \mathrm{~h}$ at room temperature. The following primary antibodies were incubated with the membrane overnight at $4^{\circ} \mathrm{C}$ :

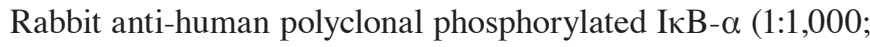
cat. no. 5209; Cell Signaling Technology, Inc., Danvers, MA, USA); rabbit anti-human polyclonal phosphorylated (p)-c-Jun N-terminal kinase (JNK; 1:1,000; cat. no. 9251; Cell Signaling Technology, Inc.); rabbit anti-human polyclonal p-p38 antibody (1:1,000; cat. no. sc-101759; Santa Cruz Biotechnology, Inc., Dallas, TX, USA); mouse anti-human monoclonal p-ERK antibody (1:1,000; cat. no. sc-7383; Santa Cruz Biotechnology, Inc.); rabbit anti-human polyclonal ERK antibody (1:1,000; cat. no. sc-94; Santa Cruz Biotechnology, Inc.); rabbit anti-human polyclonal anti- $\beta$-actin antibody (1:2,000; cat. no. sc-130656; Santa Cruz Biotechnology, Inc.); anti-iNOS (1:1,000; cat. no. ab15323; Abcam, Cambridge, MA, USA); anti-TLR2 (1:1,000; cat. no. IMG-319; Novus Biologicals, LLC., Littleton, CO, USA); and anti-TLR4 (1:1,000; cat. no. ab13556; Abcam). The membrane was then rinsed with Tris-buffered saline with Tween 20 (TBST) three times, each time for $10 \mathrm{~min}$. The membrane was then incubated with secondary horseradish peroxidase-conjugated goat 

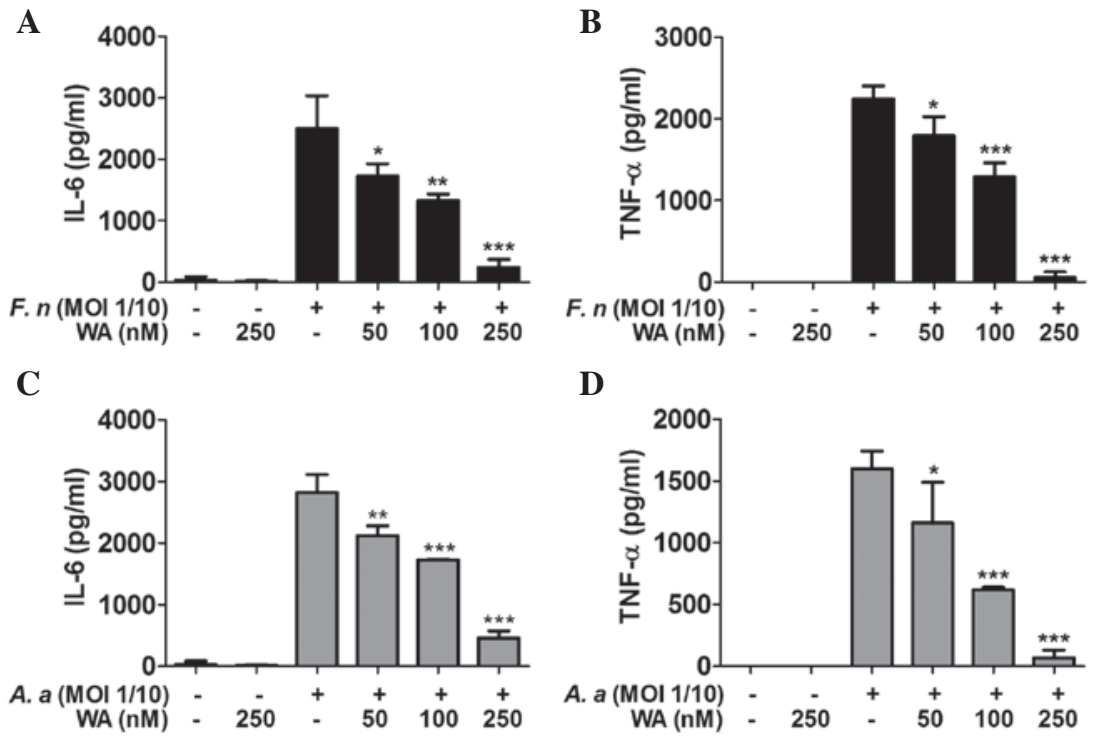

Figure 1. WA reduces the production of IL-6 and TNF- $\alpha$ by BMDMs in response to F. nucleatum and A. actinomycetemcomitans. BMDMs were infected with F. nucleatum (F. n) or A. actinomycetemcomitans (A. a) at an MOI of 10 for $6 \mathrm{~h}$ with or without pretreatment with WA (50-250 nM) for $2 \mathrm{~h}$. The concentration of (A) IL-6 and (B) TNF- $\alpha$ in culture supernatants infected with F. nucleatum, and (C) IL-6 and (D) TNF- $\alpha$ in culture supernatants infected with A. actinomycetemcomitans was determined by enzyme-linked immunosorbent assays. Data are presented as the mean \pm standard deviation of triplicate samples from one experiment representative of three independent experiments. ${ }^{*} \mathrm{P}<0.05,{ }^{* *} \mathrm{P}<0.01$ and ${ }^{* * * *} \mathrm{P}<0.001$ vs. $F$. $n(+)$ or $A . a(+)$ and $\mathrm{WA}(-)$. WA, withaferin $\mathrm{A}$; IL-6, interleukin-6; TNF- $\alpha$, tumor necrosis factor- $\alpha$; BMDM, bone marrow-derived macrophages; MOI, multiplicity of infection.

anti-rabbit IgG (1:4,000; sc-2301; Santa Cruz Biotechnology, Inc.) or goat anti-mouse IgG (1:2,000; cat. no. 2031; Santa Cruz Biotechnology, Inc.) antibodies for $2 \mathrm{~h}$ at room temperature. The membrane was then washed three time with TBST for $10 \mathrm{~min}$. Proteins were detected with SuperSignal West Pico Chemiluminescent Substrate (Gibco; Thermo Fisher Scientific, Inc.). Images of the blots were captured on CP-BU new film (Agfa HealthCare, Mortsel, Belgium) using an Automatic X-ray film processor (JP-33; JPI Healthcare, Seoul, Korea).

Statistical analysis. The differences among the mean values of different groups were assessed. Data are expressed as the mean \pm standard deviation. Statistical calculations were performed using one-way analysis of variance followed by the Tukey post test using GraphPad Prism version 5.00 (GraphPad Software, Inc., La Jolla, CA, USA). $\mathrm{P}<0.05$ was considered to indicate a statistically significant difference.

\section{Results}

WA reduces the production of $I L-6$ and TNF- $\alpha$ by macrophages in response to F. nucleatum and A. actinomycetemcomitans. To determine the inhibitory effect of WA on cytokine production by macrophages, BMDMs were pretreated with various doses of WA for $2 \mathrm{~h}$ and subsequently infected with $F$. nucleatum and A. actinomycetemcomitans for $6 \mathrm{~h}$. ELISA results showed that treatment with $F$. nucleatum and A. actinomycetemcomitans led to a substantial production of IL- 6 and TNF- $\alpha$ by the cells, which was inhibited by WA in a dose-dependent manner (Fig. 1).

$N F-\kappa B$ and MAPKactivation is inhibited by WA inmacrophages in response to $F$. nucleatum and A. actinomycetemcomitans. Our previous study demonstrated that NF- $\kappa$ B and MAPKs (p38, ERK, and JNK) are important for F. nucleatum-induced production of IL-6 and TNF- $\alpha$ (15). Induction of cytokine production by A. actinomycetemcomitans is also dependent on NF- $\mathrm{B}$ and $\mathrm{p} 38 \mathrm{MAPK}$ signaling (15). Accordingly, it was examined whether WA affects $F$. nucleatum- and A. actinomycetemcomitans-mediated activation of NF- $\kappa \mathrm{B}$ and MAPKs in BMDMs. F. nucleatum-induced phosphorylation of I $\kappa \mathrm{B}-\alpha$ and ERK was detected 15, 30, and 60 min after infection, whereas p38 and JNK were phosphorylated at 30 min post-infection (Fig. 2A). This phosphorylation of IкB- $\alpha$, p38, ERK and JNK was impaired by WA treatment (Fig. 2A). In addition, A. actinomycetemcomitans led to activation of $\mathrm{NF}-\kappa \mathrm{B}$ and MAPKs in macrophages starting $15 \mathrm{~min}$ after infection (Fig. 2B). A. actinomycetemcomitans-induced ERK phosphorylation was delayed by WA, and was only detected 60 min after infection (Fig. 2B). A. actinomycetemcomitans-induced phosphorylation of $\mathrm{I} \kappa \mathrm{B}-\alpha$ and p38 was markedly inhibited by WA treatment at all time points tested (Fig. 2B). By contrast, WA treatment only led a marginal decrease in JNK phosphorylation in macrophages in response to A.actinomycetemcomitans 30 min after infection (Fig. 2B).

WA inhibits iNOS expression and NO production in macrophages in response to $F$. nucleatum and A. actinomycetemcomitans. NO is a critical factor for the control of bacterial growth and iNOS is a key enzyme catalyzing the $\mathrm{NO}$ production from L-arginine. Periodontal pathogens can stimulate macrophages to produce NO $(19,20)$ and a selective iNOS inhibitor, mercaptoethylguanidine, prevents bone destruction in ligature-induced rodent periodontitis (21). Accordingly, it was examined whether WA had an inhibitory effect on iNOS expression and NO production induced by F. nucleatum and A. actinomycetemcomitans in macrophages. Western blot analysis demonstrated the presence of iNOS 
A

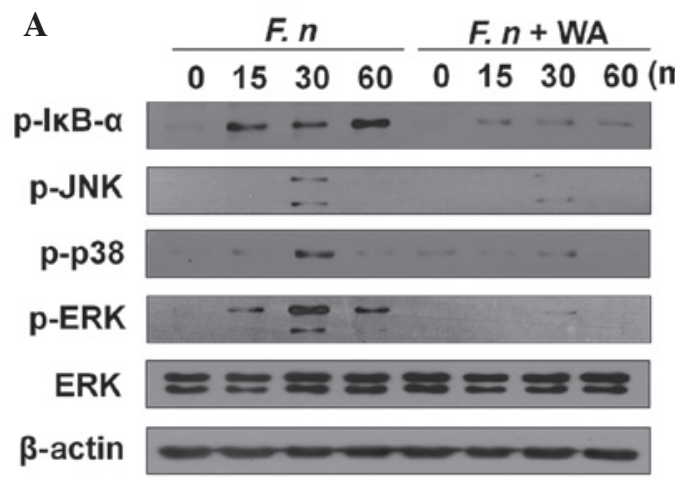

B

$$
\text { B) }
$$

p-IKB-c

p-JNK

p-p38

p-ERK

ERK

$\beta$-actin

Figure 2. WA inhibits the activation of NF-kB and MAPKs in BMDMs in response to F. nucleatum and A. actinomycetemcomitans. BMDMs were infected with (A) F.nucleatum (F. n) or (B) A. actinomycetemcomitans (A. a) at a multiplicity of infection of 10 with or without pretreatment of WA (250 nM) for $2 \mathrm{~h}$ and cellular protein was extracted at the indicated time points. Phosphorylation of IkB- $\alpha$, p38, JNK and ERK was examined by western blotting. Primary antibodies against the regular form of ERK and $\beta$-actin were used to confirm the loaded protein amounts. The results shown are from one representative experiment of two independent experiments performed. NF- $\mathrm{B}$, nuclear factor- $\mathrm{kB}$; MAPKs, mitogen-activated protein kinases; BMDMs, bone marrow-derived macrophages; WA, withaferin A; IкB- $\alpha$, JNK, c-Jun N-terminal kinases; ERK, extracellular signal-regulated kinases; p-, phosphorylated.

A

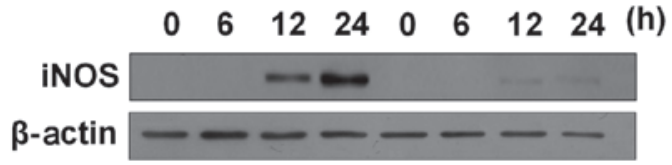

C

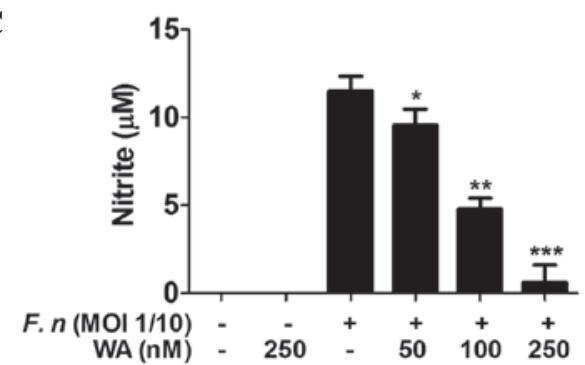

B

\begin{tabular}{|c|c|c|c|c|c|c|c|c|}
\hline & \multicolumn{4}{|c|}{ A. $a$} & \multicolumn{4}{|c|}{ A. $a+W A$} \\
\hline & 0 & 6 & 122 & 24 & 0 & 6 & 12 & 24( \\
\hline & & & -0 & - & & & - & \\
\hline & - & E & - & - & 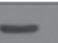 & & & \\
\hline
\end{tabular}

D

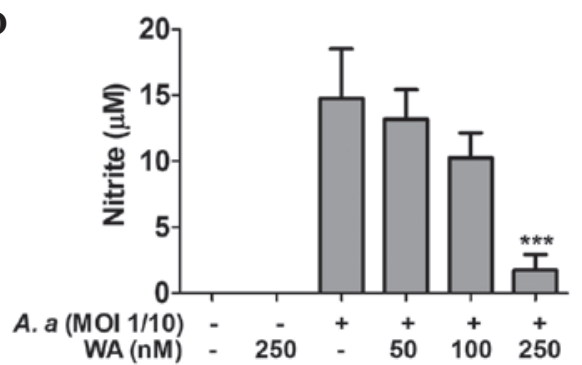

Figure 3. WA inhibits the iNOS expression and NO production induced by F. nucleatum and A. actinomycetemcomitans. BMDMs were pre-treated with and without WA (250 nM) for $2 \mathrm{~h}$, and then infected with (A) F. nucleatum (F. n) or (B) A. actinomycetemcomitans (A. a) at an MOI of 10 for the indicated time points. The cellular proteins were harvested at $24 \mathrm{~h}$ and the amounts of (A and B) iNOS was determined by western blot analysis. As a separate experiment, the cells were pre-treated with or without WA $(50-250 \mathrm{nM})$ and infected with $F . n$ or $A . a$ at an MOI of 10 for $24 \mathrm{~h}$. (C and D) NO production was measured by the Griess reaction. The results are from one representative experiment of three independent experiments. ${ }^{*} \mathrm{P}<0.05,{ }^{* * *} \mathrm{P}<0.01,{ }^{* * *} \mathrm{P}<0.001$ vs. $F$. $n(+)$ or $A . a(+)$ and WA (-). iNOS, inducible nitric oxide synthase; WA, withaferin A; BMDMs, bone marrow-derived macrophages; MOI, multiplicity of infection.

protein in $F$. nucleatum-infected macrophages 12 and $24 \mathrm{~h}$ after infection, which was mostly impaired by WA treatment (Fig. 3A). A. actinomycetemcomitans also induced iNOS expression with similar kinetics to F. nucleatum (Fig. 3B). WA inhibited A. actinomycetemcomitans-induced expression of iNOS at $24 \mathrm{~h}$, while it had little effect on iNOS expression at $12 \mathrm{~h}$ (Fig. 3B). The level of NO in the culture supernatants of BMDMs infected with F. nucleatum or A. actinomycetemcomitans was detected in the absence or presence of WA. However, NO was undetectable under these conditions regardless of bacterial infection or WA treatment (data not shown), even though $F$. nucleatum and A. actinomycetemcomitans could induce expression of iNOS in macrophages. Therefore, the experimental design was altered and cells were also treated with interferon- $\gamma$, which is known to enhance NO production in macrophages (22). The results showed that $F$. nucleatum and A. actinomycetemcomitans induced NO production in
BMDMs, which was inhibited by WA in a dose-dependent manner (Fig. 3C and D). These findings indicate that WA may effectively inhibit NO production induced by periodontal pathogens in macrophages.

WA reduces the expression of TLR2 and TLR4 in macrophages in response to $F$. nucleatum and A. actinomycetemcomitans. TLR2 and TLR4 are involved in the production of IL- 6 and TNF- $\alpha$ in macrophages in response to $F$. nucleatum and A. actinomycetemcomitans (15). Therefore, this study aimed to determine whether WA affects the expression of TLR2 and TLR4 in macrophages. F. nucleatum increased the protein expression of TLR 2 and TLR4 in macrophages $6 \mathrm{~h}$ after infection, and the TLR 2 expression level remained increased at $24 \mathrm{~h}$ (Fig. 4A). Notably, WA reduced the endogenous and induced expression of TLR2 and TLR4 in F. nucleatum-infected macrophages (Fig. 4A). A. actinomycetemcomitans also 
A

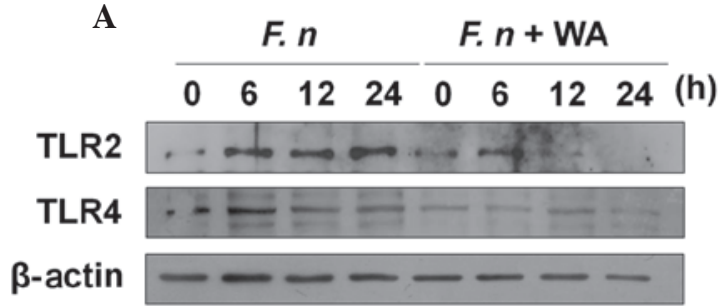

B

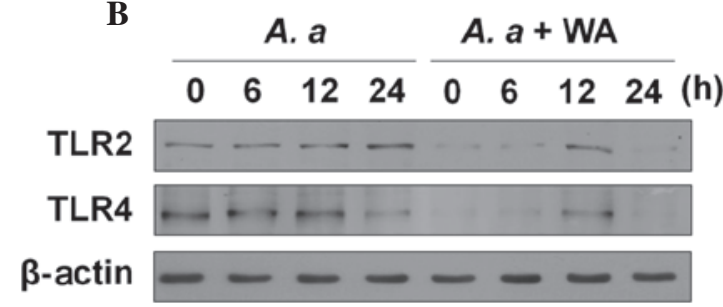

Figure 4. WA reduces the expression of TLR2 and TLR4 in BMDMs in response to F. nucleatum and A. actinomycetemcomitans. BMDMs were infected with (A) F.nucleatum (F. n) or (B) A. actinomycetemcomitans (A. a) at a multiplicity of infection of 10 with or without pretreatment of WA (250 nM) for $2 \mathrm{~h}$ and cellular protein was extracted at the indicated time points. The expression of TLR 2 and TLR 4 was examined by western blotting. $\beta$-actin served as a loading control. The results are from one representative experiment of two independent experiments performed. TLR, toll-like receptor; BMDMs, bone marrow-derived macrophages; WA, withaferin A.

marginally increased the TLR 2 expression in macrophages at 12 and $24 \mathrm{~h}$ after infection, whereas the TLR4 expression level was decreased in the cells at $24 \mathrm{~h}$ (Fig. 4B). Likewise, WA inhibited both TLR2 and TLR4 expression in A. actinomycetemcomitans-infected macrophages (Fig. 4B). These findings suggest that WA may exert its anti-inflammatory effect in macrophages in response to $F$. nucleatum and A. actinomycetemcomitans by inhibiting TLR-mediated signaling.

\section{Discussion}

Periodontal destruction is the result of the complex interplay between pathogenic bacteria and the host immune responses (23). Bacterial components, such as LPS, and toxic products of periodontal pathogens trigger host immune responses, which results in the destruction of the periodontal tissue. Traditional therapeutics controlling periodontal diseases include dental cleaning, subgingival scaling/root planning and the use of antibiotics. These treatments can reduce the levels of pathogenic bacteria in periodontal pockets (24). In addition to the use of antibiotics, there has been an increased interest in the use of natural products as adjuncts to manage inflammatory disorders, including periodontitis, due to their anti-inflammatory properties (6).

In the present study, the anti-inflammatory effects of WA on F. nucleatum- and A. actinomycetemcomitans-infected macrophages were investigated. The results showed that WA has inhibitory effects on cytokine production, the activation of NF- $\kappa \mathrm{B}$ and MAPKs, and NO production in macrophages in response to the two types of bacteria. This suggests that WA can be used as a natural preventive and therapeutic agent for periodontitis. It is well-known that WA has NF- $\mathrm{B}$ inhibiting activity in a wide range of cell types in response to various stimuli (7). However, the effect of WA on MAPK activation appears to be dependent on the cell type and stimuli. WA alone has been shown to activate p38, ERK and JNK MAPKs in U937 human leukemic cells with different kinetics (25). In addition, WA was shown to induce the phosphorylation of p38 in MCF-7 breast cancer cells within $3 \mathrm{~h}$ of treatment (26). In K562 human erythromyeloblastoid leukemia cells, WA induced activation of ERK, although p38 activation was not affected (27). By contrast, WA inhibited TNF- $\alpha$-induced activation of ERK in human pulmonary epithelial cells, although it did not alter the activation of p38 and JNK (28). In the present study, WA could almost completely inhibit the activation of $\mathrm{NF}-\kappa \mathrm{B}$ and
MAPKs in macrophages in response to $F$. nucleatum and $A$. actinomycetemcomitans, although its inhibitory effect on $A$. actinomycetemcomitans-induced JNK phosphorylation was not identified to be significant. It remains to be elucidated whether the inhibitory effect of WA on MAPKs is specific to periodontal pathogens and/or macrophages.

TLR2 and TLR4 appears to be critical for periodontal pathogen-induced immune responses although there is controversy regarding the bacterial preparation used and the cell type tested (14-16). In a study using sonicated bacteria, A. actinomycetemcomitans induced IL-8 production in HEK293 cells via TLR2 and TLR4, whereas $F$. nucleatum elicited IL-8 production exclusively via a TLR2-dependent pathway (14). In human periodontal ligament cells, TLR2 and TLR4 are essential for the F. nucleatum-induced production of cytokines (16). Likewise, in macrophages, double deficiency of TLR2 and TLR4 leads to decreased production of IL- 6 and TNF- $\alpha$, and delayed $\mathrm{I} \kappa \mathrm{B}-\alpha$ degradation in response to $F$. nucleatum and A. actinomycetemcomitans (15). In the present study, it was demonstrated for the first time that WA inhibits endogenous and induced expression of TLR2 and TLR4 in macrophages, indicating that WA can regulate TLR-mediated signaling. In fact, in the present study, an MOI of $1 / 10$ was used to stimulate macrophages. This dose of F. nucleatum and A. actinomycetemcomitans could induce the production of substantial levels of TNF- $\alpha$ in TLR2/4 double-deficient macrophages (15) and a high dose of WA (250 nM) completely inhibited TNF- $\alpha$ production in this study. This suggests that WA may inhibit other signaling proteins involved in F. nucleatum and A. actinomycetemcomitans-induced production of cytokines in addition to TLR2 and TLR4. Previously it was demonstrated that endosomal TLRs are important for cytokine production in TLR2/4 double-deficient macrophages (15), suggesting that WA likely exerts its anti-inflammatory effects by direct inhibition of multiple TLRs. In addition to TLRs, Nod-like receptors (NLRs) participate in the bacteria-induced immune responses in host cells. The first identified NLRs, Nod1 and Nod2, recognize the peptidoglycan motifs meso-diaminophimelic acid and muramyl dipeptide, respectively (29). Periodontal pathogens, including $F$. nucleatum and A. actinomycetemcomitans, are known to stimulate Nod1- and Nod2, and their peptidoglycans induce NF- $\kappa$ B activation in Nod1- and Nod2-transfected HEK293 cells (30). A previous study revealed that vimentin, an intermediate filament protein, is an important regulator of Nod2 function (31). WA has been shown to disrupt 
the interaction between vimentin and Nod2, and inhibit Nod2-dependent NF- $\kappa B$ activation (31). Although the role of Nod2 in the cytokine production by macrophages in response to $F$. nucleatum and A. actinomycetemcomitans remains unknown, it would be useful to clarify whether WA regulates the Nod2-mediated immune response in macrophages in response to periodontal pathogens.

In conclusion, it was demonstrated that WA has inhibitory effects on F. nucleatum- and A. actinomycetemcomitans-induced immune responses in macrophages by downregulating TLR signaling. These results indicate that WA may have potential as a novel therapeutic and preventive agent for periodontitis.

\section{Acknowledgements}

This study was supported by the Basic Science Research Program through the National Research Foundation of Korea (NRF) funded by the Ministry of Science ICT \& Future Planning (grant nos. 2012R1A1A2041944 and 2014R1A4A1005309).

\section{References}

1. Costalonga M and Herzberg MC: The oral microbiome and the immunobiology of periodontal disease and caries. Immunol Lett 162: 22-38, 2014.

2. Gjermo P, Rösing CK, Susin C and Oppermann R: Periodontal diseases in Central and South America. Periodontol 2000 29: 70-78, 2002.

3. Albandar JM: Epidemiology and risk factors of periodontal diseases. Dent Clin North Am 49: 517-532, v-vi, 2005.

4. Jotwani R and Cutler CW: Adult periodontitis - specific bacterial infection or chronic inflammation? J Med Microbiol 47: 187-188, 1998.

5. Darveau RP, Tanner A and Page RC: The microbial challenge in periodontitis. Periodontology 2000 14: 12-32, 1997.

6. Palaska I, Papathanasiou E and Theoharides TC: Use of polyphenols in periodontal inflammation. Eur J Pharmacol 720: 77-83, 2013.

7. Vanden Berghe W, Sabbe L, Kaileh M, Haegeman G and Heyninck $\mathrm{K}$ : Molecular insight in the multifunctional activities of Withaferin A. Biochem Pharmacol 84: 1282-1291, 2012.

8. Oh JH, Lee TJ, Park JW and Kwon TK: Withaferin A inhibits iNOS expression and nitric oxide production by Akt inactivation and down-regulating LPS-induced activity of NF-kappaB in RAW 264.7 cells. Eur J Pharmacol 599: 11-17, 2008.

9. Maitra R, Porter MA, Huang S and Gilmour BP: Inhibition of NFkappaB by the natural product Withaferin A in cellular models of Cystic Fibrosis inflammation. J Inflamm (Lond) 6: 15, 2009.

10. Mohan R, Hammers HJ, Bargagna-Mohan P, Zhan XH, Herbstritt CJ, Ruiz A, Zhang L, Hanson AD, Conner BP, Rougas J and Pribluda VS: Withaferin A is a potent inhibitor of angiogenesis. Angiogenesis 7: 115-122, 2004.

11. Vyas AR and Singh SV: Molecular targets and mechanisms of cancer prevention and treatment by withaferin a, a naturally occurring steroidal lactone. AAPS J 16: 1-10, 2014.

12. Hahm ER and Singh SV: Withaferin A-induced apoptosis in human breast cancer cells is associated with suppression of inhibitor of apoptosis family protein expression. Cancer Lett 334: 101-108, 2012.

13. Kawai T and Akira S: The role of pattern-recognition receptors in innate immunity: update on Toll-like receptors. Nat Immunol 11: 373-384, 2010.
14. Kikkert R, Laine ML, Aarden LA and van Winkelhoff AJ: Activation of toll-like receptors 2 and 4 by gram-negative periodontal bacteria. Oral Microbiol Immunol 22: 145-151, 2007.

15. Park SR, Kim DJ, Han SH, Kang MJ, Lee JY, Jeong YJ, Lee SJ, Kim TH, Ahn SG, Yoon JH and Park JH: Diverse Toll-like receptors mediate cytokine production by Fusobacterium nucleatum and Aggregatibacter actinomycetemcomitans in macrophages. Infect Immun 82: 1914-1920, 2014.

16. Sun Y, Shu R, Li CL and Zhang MZ: Gram-negative periodontal bacteria induce the activation of Toll-like receptors 2 and 4 , and cytokine production in human periodontal ligament cells. J Periodontol 81: 1488-1496, 2010.

17. Celada A GP, Rinderknecht E, Schreiber RD: Evidence for a gamma-interferon receptor that regulates macrophage tumoricidal activity. J Exp Med 160: 55-74, 1984.

18. Green LC, Wagner DA, Glogowski J, Skipper PL, Wishnok JS and Tannenbaum SR: Analysis of nitrate, nitrite, and [15N] nitrate in biological fluids. Anal Biochem 126: 131-138, 1982.

19. Frolov I, Houri-Hadad Y, Soskolne A and Shapira L: In vivo exposure to Porphyromonas gingivalis up-regulates nitric oxide but suppresses tumour necrosis factor-alpha production by cultured macrophages. Immunology 93: 323-328, 1998.

20. Blix IJ and Helgeland K: LPS from Actinobacillus actinomycetemcomitans and production of nitric oxide in murine macrophages J774. Eur J Oral Sci 106: 576-581, 1998.

21. Lohinai Z, Benedek P, Fehér E, Györfi A, Rosivall L, Fazekas A, Salzman AL and Szabó C: Protective effects of mercaptoethylguanidine, a selective inhibitor of inducible nitric oxide synthase, in ligature-induced periodontitis in the rat. Brit J Pharmacol 123: 353-360, 1998.

22. Totemeyer S, Sheppard M, Lloyd A, Roper D, Dowson C, Underhill D, Murray P, Maskell D and Bryant C: IFN-gamma enhances production of nitric oxide from macrophages via a mechanism that depends on nucleotide oligomerization domain-2. J Immunol 176: 4804-4810, 2006.

23. Benakanakere $M$ and Kinane DF: Innate cellular responses to the periodontal biofilm. Front Oral Biol 15: 41-55, 2012.

24. Heitz-Mayfield LJ, Trombelli L, Heitz F, Needleman I and Moles D: A systematic review of the effect of surgical debridement vs non-surgical debridement for the treatment of chronic periodontitis. J Clin Periodontol 29 (Suppl 3): 92-102; discussion 160-162, 2002.

25. Oh JH, Lee TJ, Kim SH, Choi YH, Lee SH, Lee JM, Kim YH, Park JW and Kwon TK: Induction of apoptosis by withaferin A in human leukemia U937 cells through down-regulation of Akt phosphorylation. Apoptosis 13: 1494-1504, 2008.

26. Zhang X, Mukerji R, Samadi AK and Cohen MS: Down-regulation of estrogen receptor-alpha and rearranged during transfection tyrosine kinase is associated with withaferin a-induced apoptosis in MCF-7 breast cancer cells. BMC Complement Altern Med 11: 84, 2011.

27. Suttana W, Mankhetkorn S, Poompimon W, Palagani A, Zhokhov S, Gerlo S, Haegeman G and Berghe WV: Differential chemosensitization of P-glycoprotein overexpressing K562/Adr cells by withaferin A and Siamois polyphenols. Mol Cancer 9: 99, 2010.

28. Oh JH and Kwon TK: Withaferin A inhibits tumor necrosis factor-alpha-induced expression of cell adhesion molecules by inactivation of Akt and NF-kappaB in human pulmonary epithelial cells. Int Immunopharmacol 9: 614-619, 2009.

29. Caruso R, Warner N, Inohara N and Núñez G: NOD1 and NOD2: Signaling, host defense, and inflammatory disease. Immunity 41: 898-908, 2014.

30. Okugawa T, Kaneko T, Yoshimura A, Silverman N and Hara Y: NOD1 and NOD2 mediate sensing of periodontal pathogens. J Dent Res 89: 186-191, 2010.

31. Stevens C, Henderson P, Nimmo ER, Soares DC, Dogan B, Simpson KW, Barrett JC; International Inflammatory Bowel Disease Genetics Consortium, Wilson DC and Satsangi J: The intermediate filament protein, vimentin, is a regulator of NOD2 activity. Gut 62: 695-707, 2013. 